\title{
The forest fire prediction in JiangXi province based on PSO-GA-BP neural network
}

\author{
Zhijian Yin ${ }^{1, a}$, Fan Wang ${ }^{1, b}$, Tiantian Tang ${ }^{1, c}$, Qiang Luo ${ }^{1, d}$ and Kun Xiang ${ }^{1, e}$ \\ ${ }^{1}$ Department of Communication and Electronic, Jiangxi Science \& Technology Normal University, \\ Nanchang, 330013, China \\ aemail: 86412656@qq.com, bemail: 1245905084@qq.com, cemail: 614994205@qq.com, \\ demail:1060536061@qq.com, eemail: 1244689313@qq.com
}

Keywords: Forest fire danger rating; Meteorological factors; PSO-GA-BP neural network;

\begin{abstract}
In this paper, we collected the meteorological data and forest fire danger rating data of four stations in JiangXi (NanChang, JingDeZhen, GiAn, GanZhou) from 2013 to 2015, and build a neural network fire prediction model. Then use GA, PSO and PSO-GA hybrid algorithm to improve BP neural network.By contrasting the prediction results of BP network, GA-BP network, PSO-BP network and PSO-GA-BP network, the prediction accuracy of PSO-GA-BP network is the highest. The result of experiment shows that the effect of BP network optimized by PSO-GA is the best, compared with GA and PSO.
\end{abstract}

\section{Introduction}

The forest has abundant natural resources, the development of human society relies on the abundant resources of the forest. However, forest fires became one of the most serious natural disasters, it seriously threat to the existing forest resources. Therefore, the forest fire prediction has great significance for the development of human society, the protection of forest resources and ecological environment.

According to the data of Chinese bureau, from 2013 to 2004, the forest fire incidence in China is 86609 , the total fire area is 1986127.27 hectares, the total affected forest area is 852558.59 hectares, the loss caused by fire is about 1.29 billion yuan. However, the forest fire incidence in JiangXi is 3254 accounting for $3.76 \%$ of the national forest fire, the total affected forest area is 34025.88 hectares $(3.99 \%)$, the caused economic loss is about 102.8 million yuan accounting $(7.98 \%)$, the total number of casualties are 102 , including death toll of 34. all kinds of fire data in JiangXi is located in the national top 10 , and the losses caused by fire are huge.

Meteorological factor has been the important influence factors of forest fires. Many researchers use meteorological factor to build artificial neural network fire prediction model, and have some achievements. So the neural network fire prediction model can provide early warning for forest fire prevention work, and be good for forest fire prevention work.

\section{The basic principle of neural network}

Artificial neural network is a simulation of human brain neural network. We know that the brain is made of a large number of biological neurons, it is complex and has the function of processing and transmitting information.

\section{The BP neural network}

BP neural network is a kind of multilayer neural network trained by error back propagation algorithm, it greatly promoted the development of the neural network and the most widely used at present. BP neural network is composed of input layer, output layer and hidden layer, the hidden layer can be a layer or multilayer. As shown in figure 1, the neurons of three layers are linked by the weight $\mathrm{W}$, but at the same layer neurons are not connected.

BP algorithm includes two processes, forward and back transmission. When input samples to BP network, it need forward transmission to get the output of all the network layer. When output layer get the actual output, we can calculate the error compared with the desired output. Then, the 
error is back transmitted to adjust weights and thresholds of each layer. Repeat the two processes to reduce the error of the output layer, until it no longer changed.

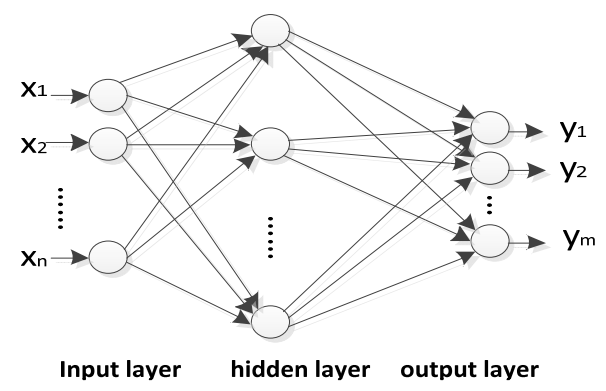

Fig.1. Structure of BP neural network with single hidden layer

\section{Genetic Algorithm (GA)}

Genetic algorithm is an intelligent algorithm to imitate biological evolution and genetic principle. It has three operations (selection, crossover and mutation).

(1) Selection

Selection means choosing the individuals with high adaptability from groups to form new population. The selection of GA commonly uses the roulette wheel method, its mathematical expressions is shown below.

$$
P_{i}=\frac{F_{i}}{\sum_{i=1}^{n} F_{i}}
$$

Among them, Pi means the probability to be selected of the individual, Fi is individual fitness value, $\mathrm{n}$ is the total number of individuals in the population.

(2) Crossover

Crossover means two individuals exchange their chromosomes to form new chromosomes. GA has the crossover probability which means the probability to crossover of the selected individuals.

(3) Mutation

Mutation is a kind of the genetic gene mutations, and is that the an individual chromosome has mutated to be a new individual. GA also has mutation probability, and it is very small.

\section{Particle swarm optimization algorithm (PSO)}

PSO is an algorithm to simulate birds feed. The position and velocity represent the characteristics of the particles in the algorithm, in addition, the fitness is used to judge good or bad of the particles. And particles will update their location and velocity based on two extreme value, one is individual extreme value (Pbest), the other is group extreme value (Gbest).

Assuming the dimension of searching space of the particle population is $\mathrm{D}$, the number of particles in population $\mathrm{X}$ is $\mathrm{n}, X=\left(X_{1}, X_{2}, \Lambda, X_{n}\right) . \mathrm{Xi}$ is the ith particle's position, $X_{i}=\left(x_{i 1}, x_{i 2}, \Lambda, x_{i D}\right)^{T}$. The velocity of the ith particle: $V_{i}=\left(V_{i 1}, V_{i 2}, \Lambda, V_{i D}\right)^{T}$. The Pbest of the ith particle: $P_{i}=\left(P_{i 1}, P_{i 2}, \Lambda, P_{i D}\right)^{T}$. The Gbest: $P_{g}=\left(P_{g 1}, P_{g 2}, \Lambda, P_{g D}\right)^{T}$.

In the iteration process of PSO, the updating formula of particle velocity and position is shown below.

$$
\begin{aligned}
& V_{i d}^{k+1}=w V_{i d}^{k}+c_{1} r_{1}\left(P_{i d}^{k}-X_{i d}^{k}\right)+c_{2} r_{2}\left(P_{g d}^{k}-X_{g d}^{k}\right) \\
& X_{i d}^{k+1}=X_{i d}^{k}+V_{i d}^{k+1}
\end{aligned}
$$

\section{Build neural network prediction model}

\section{The design of the neural network structure}

In this paper, the topology of the network is 13-16-1. Input layer has 13 neurons, it depends on the number of meteorological factors, the 13 meteorological factors are shown in table1. The neurons number of hidden layer is 16 , it is always determined by empirical formula and 
experiments. According to the theory of Hecht-Nielsen, network with single hidden layer can realize arbitrary function, so there is only one hidden layer. The output layer has one neuron which is the forest fire danger rating.

Tab.1. Part of the experimental sample data

\begin{tabular}{|c|c|c|c|c|c|c|c|c|c|c|c|c|c|c|}
\hline 荧 & 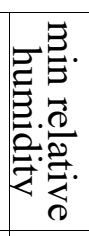 & 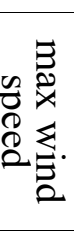 & 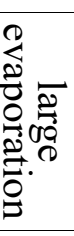 & 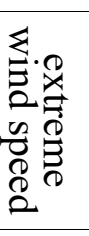 & 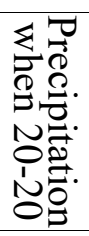 & 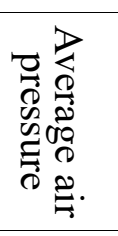 & 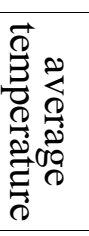 & 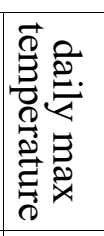 & 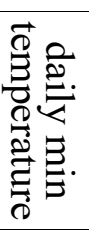 & 局距 & 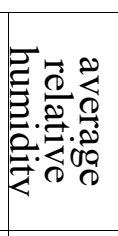 & 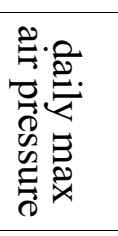 & 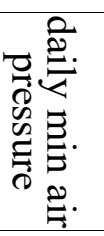 & 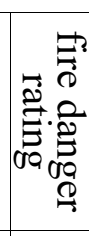 \\
\hline 1 & 69 & 39 & 18 & 84 & 108 & 9999 & 262 & 314 & 226 & 6 & 91 & 10016 & 9975 & 1 \\
\hline 2 & 56 & 47 & 32 & 93 & 0 & 9970 & 293 & 344 & 243 & 90 & 78 & 9998 & 9940 & 3 \\
\hline 3 & 62 & 60 & 36 & 118 & 0 & 9936 & 295 & 327 & 269 & 10 & 70 & 9954 & 9918 & 2 \\
\hline 4 & 75 & 27 & 19 & 56 & 332 & 9961 & 242 & 276 & 226 & 0 & 93 & 9981 & 9936 & 1 \\
\hline 5 & 66 & 25 & 26 & 41 & 0 & 9998 & 251 & 284 & 225 & 0 & 85 & 10007 & 9981 & 2 \\
\hline 6 & 54 & 33 & 25 & 55 & 2 & 10002 & 270 & 325 & 234 & 73 & 82 & 10018 & 9984 & 2 \\
\hline 7 & 81 & 50 & 33 & 93 & 175 & 9998 & 238 & 273 & 221 & 0 & 94 & 10007 & 9982 & 1 \\
\hline 8 & 84 & 24 & 19 & 66 & 622 & 9988 & 228 & 274 & 210 & 0 & 99 & 10002 & 9971 & 1 \\
\hline 9 & 58 & 47 & 28 & 76 & 0 & 9970 & 303 & 344 & 260 & 99 & 77 & 9987 & 9949 & 3 \\
\hline 10 & 56 & 55 & 45 & 119 & 0 & 9948 & 311 & 349 & 281 & 89 & 70 & 9966 & 9925 & 3 \\
\hline
\end{tabular}

\section{Experimental data processing}

In this paper, we have collected 1017 sets of experimental sample data with three forest fire danger rating classes, and each class has 339 groups of data. The data of BP contains the training data and testing data, there are 960 groups of data (320 for each class) for training, and 57 for testing.

The dimension of each meteorological factors is different, it will lead to different dispersion degree of the variables. So the original data need to be standardized, it can eliminate the effects caused by the different dimensions of data, and improve the accuracy of network computing. Before the prediction of network, we usually adopt data normalization method to deal with sample data, in order to transform it into the number between $[0,1]$.

\section{The steps and processs of PSO-GA}

GA and PSO are used to optimize BP by optimizing its connection weights and threshold. In this paper, we mix GA and PSO, this algorithm adds GA into PSO. This hybrid algorithm combines their advantages, with less amount of calculation, fast convergence and good global convergence. The implementation steps of PSO-GA algorithm are shown in figure 2.

(1) Initialize the particle population. Inludes the particle's position and velocity, and some parameters.

(2) Calculate the particle's fitness value and find Pbest and Gbest. The expression of the fitness function: fitness $=$ mse $($ an - outputn $)$. Mse is a function of mean square error calculation in matlab. It means that the fitness value in this paper is the mean square error between prediction value and expectation of BP network output. The smaller the fitness value is, the smaller the error, the higher the prediction accuracy of the neural network. So the smaller fitness is the best value.

(3) Update the particle's position, speed and fitness value.

(4) Order the fitness value from small to large, and divide it into two equal parts. The particle population with smaller fitness value is defined as P1, another is P2. 
(5) Add the genetic algorithm. Process the selection and crossover for P2.

(6) Order the fitness value again, and divide it into two equal parts (P3 and P4).

(7) Process the mutation for P4.

(8) Update Pbest and Gbest. Compare the particle's fitness value, the particle with the smallest value is the best.

(9) The end. When the number of iterations has been the setting maximum, or the fitness value has been better, the algorithm ends. Otherwise, return to step 2 .

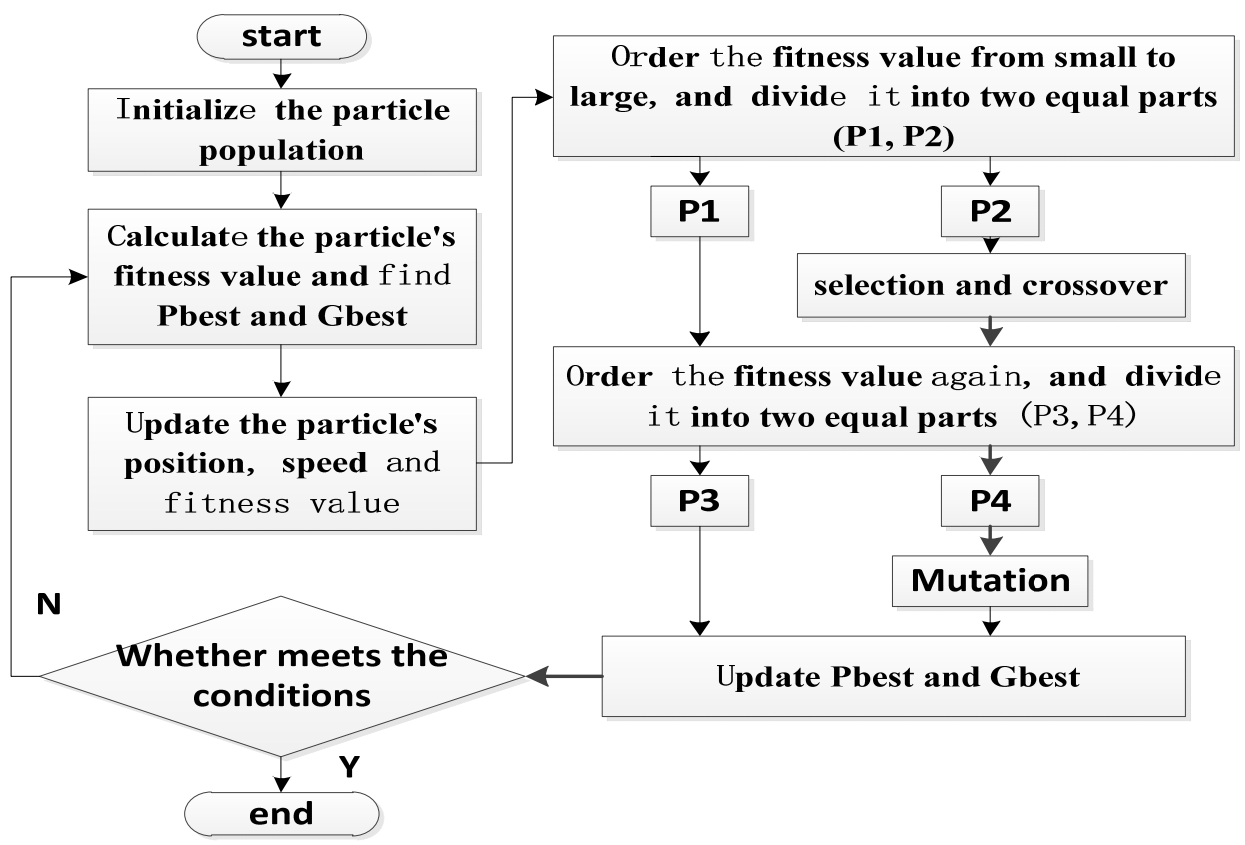

Fig.2. The implementation steps of PSO-GA

\section{The MATLAB simulation results}

The simulation platform is matlab R2011b, we use the function newff of neural network toolbox to realize the construction, training and simulation of BP network. In this paper, there are four kinds of forest fire prediction model. The experimental results of the four models are shown in figure 3 .

The prediction accuracy of four networks is shown in table 2 . We can see that, the prediction accuracy of PSO-GA-BP network model is $80.70 \%$, the PSO-BP model is $75.44 \%$, the GA-BP network model is $73.68 \%$, the BP model without optimization is $70.18 \%$. Among the four network model, the prediction accuracy of PSO-GA-BP network model is the highest. The prediction effect of PSO-GA-BP model is the best, the result shows that the mixture of PSO and GA algorithm can optimize BP network with better effects.

Tab.2. The prediction accuracy of four models

\begin{tabular}{|c|c|c|c|c|}
\hline Forest fire prediction model & Prediction accuracy & First class & Second class & Third class \\
\hline BP network & 0.7018 & 0.7368 & 0.7368 & 0.6316 \\
\hline GA-BP network & 0.7368 & 0.8421 & 0.7895 & 0.5789 \\
\hline PSO-BP network & 0.7544 & 0.7368 & 0.6316 & 0.8947 \\
\hline PSO-GA-BP network & 0.8070 & 0.8947 & 0.7895 & 0.7368 \\
\hline
\end{tabular}



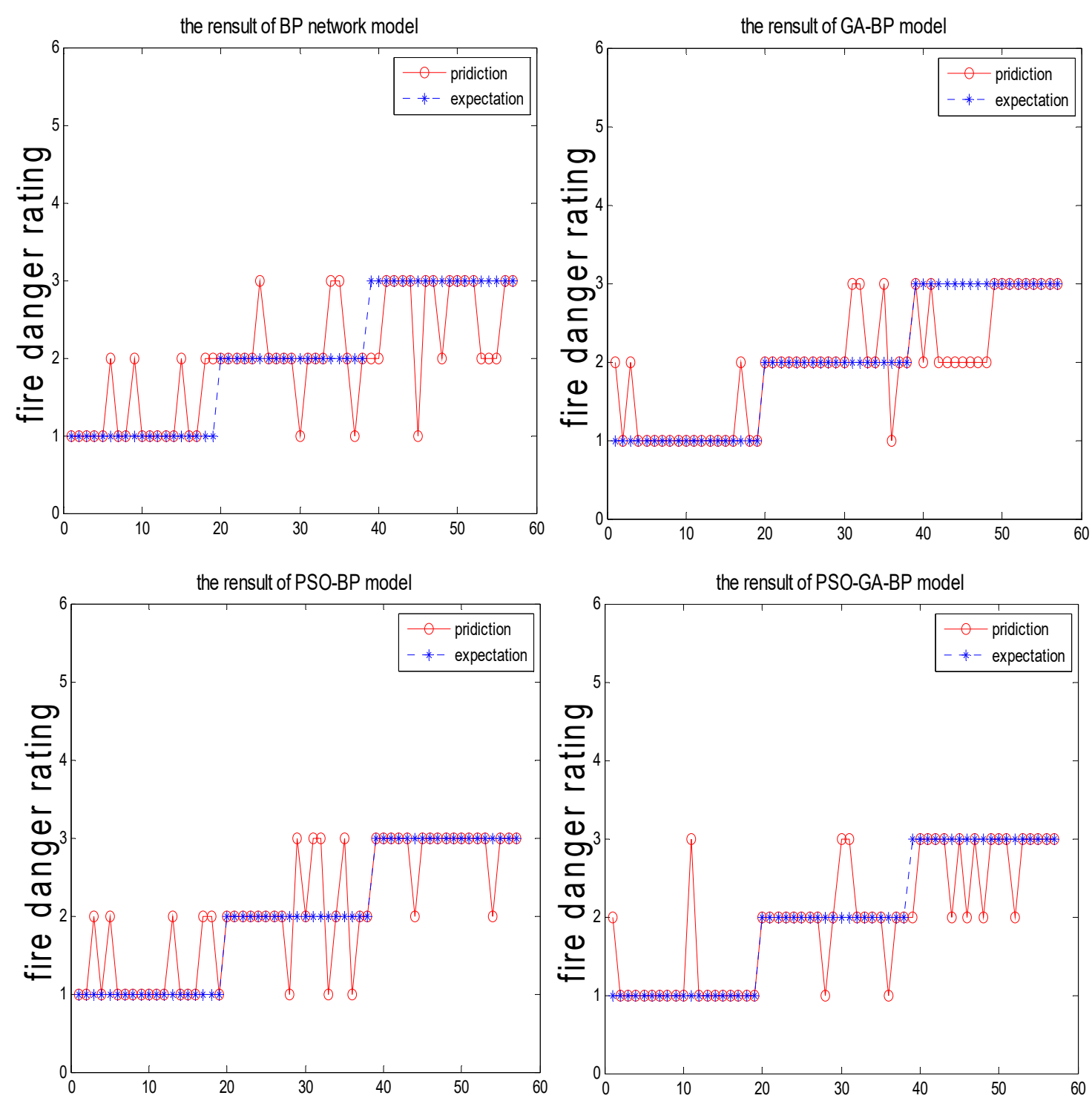

Fig.3. the experimental results of four models

\section{References}

[1] Dandan Wang, Jiarong Huang, Wei Liu, etc. The forest fire danger prediction based on artificial neural network[J]. Journal of northwest forestry college. 2010, 25(3):143-146.

[2] Zhangwen Su, Aiqin Liu, Huiling Liang, etc. The forest fire danger forecasting model of FuJian based on the meteorological factors[J]. Journal of forest and environment. 2015,35(4):370-376.

[3] Zhaolou Qian. A kind of prediction method based on BP neural network optimized by improved PSO [J]. Journal of electronic test. 2015,20:39-40.

[4] Pan Wang, Jian Chen, Zhonghua Cao, etc. The predictive model of comprehensive agricultural mechanization level based on GA-BP neural network[J]. Journal of agricultural research. 2016(3):75-79.

[5] Yanan Wang, Xiaojing Meng. Based on neural network with momentum BP algorithm to predict the housing price[J]. Software Guide. 2015,14(2):59-61.

[6] Yuming Gao. The short-term power load prediction based on the BP network optimized by GA-PSO[D]. GuiZhou normal university, 2014. 